\title{
Institucionalidad y autonomía en la práctica lectora de docentes de secundaria que laboran en centros educativos de las provincias de Heredia y Cartago
}

\author{
Gilbert Ulloa Brenes' ${ }^{1}$ Andrea Rojas Vargas² \& Ilse Gutiérrez Schwanhäuser ${ }^{3}$
}

1. Profesor e investigador del Centro de Investigaciones en Educación, Escuela de Ciencias de la Educación, UNED. San José, Costa Rica; gulloa@uned.ac.cr

2. Investigadora del Centro de Investigaciones en Educación, Escuela de Ciencias de la Educación, UNED. San José, Costa Rica; anrojasv@uned.ac.cr

3. Encargada de la Carrera de Docencia, Escuela de Ciencias de la Educación, UNED.San José, Costa Rica; igutierrez@uned.ac.cr

\section{Resumen}

A partir de los resultados del proyecto Construcción de un modelo interpretativo sobre la lectura como práctica social en docentes de colegios académicos y técnicos, diurnos y nocturnos de las provincias de Cartago y Heredia, este artículo tiene por objetivo discutir sobre las relaciones entre la lectura como obligación institucional y la lectura como gusto propio que apuntaría a una actividad autónoma, en la práctica lectora de ocho docentes de secundaria, quienes laboran en instituciones educativas rurales y urbanas de las provincias de Heredia y Cartago. Como fundamento analítico se parte de la propuesta elaborada por Ulloa (2017) y la discusión de resultados se basa en las tesis de Bourdieu (2007; 2010) respecto de las prácticas sociales. Los datos analizados se recolectaron a través de un proceso de investigación cualitativa en el que se realizaron ocho entrevistas en profundidad, de tipo biográfico-narrativo, con cuatro docentes que laboran en colegios de las provincias de Heredia y Cartago. Los principales hallazgos evidencian que, si bien estos docentes tienden a mirar con recelo la obligatoriedad en la práctica de la lectura, oponiéndola a aspectos como el placer y el conocimiento que derivan de la lectura por gusto propio, buena parte de su actividad lectora se basa en el cumplimiento de esa obligatoriedad que adquiere la forma de lecturas enfocadas en su trabajo.

Palabras clave: Lectura, educación secundaria, docencia, instituciones culturales, investigación cualitativa.

\section{Abstract \\ Institutionality and autonomy in the reading practice of secondary teachers that work in educational centers of the provinces of Heredia and Cartago}

Based on the results of the Project Construcción de un modelo interpretativo sobre la lectura como práctica social en docentes de colegios académicos y técnicos, diurnos y nocturnos de las provincias de Cartago y Heredia, this article aims to discuss the relationship between reading as an institutional obligation and reading as a personal pleasure that would point to an autonomous activity, in the reading practice of eight high school teachers, who work in rural and urban educational institutions in the provinces of Heredia and Cartago. The analytical foundation is based on the proposal developed by Ulloa (2017) and the discussion of results is based on the theses of Bourdieu $(2007,2010)$ regarding social practices. 
The data analyzed were collected through a qualitative research process in which eight in-depth interviews were conducted, biographical-narrative, with four teachers working in schools in the provinces of Heredia and Cartago.

The main findings show that although these teachers tend to look with distrust on the practice of reading, opposing aspects such as pleasure and knowledge derived from reading for their own pleasure, a good part of their reading activity is based on the fulfillment of this obligation that acquires the form of the consumption of readings focused on their work.

Key words: Reading, secondary education, teaching, cultural institutions, qualitative research.

\section{INTRODUCCIÓN}

La problematización y abordaje metodológico del tema que se expone en este artículo deriva de dos circunstancias, una de orden social y otra de filiación académica.

En primera instancia, el interés por estudiar la lectura como una práctica circunscrita por las características sociales del contexto educativo, se inscribe en una serie de preocupaciones que, de diferente manera y desde diferentes actores sociales, cuestiona la forma como hoy se configuran las prácticas lectoras y el papel que cumplen instancias gubernamentales como el Ministerio de Cultura y Juventud o el Ministerio de Educación Pública en su apuntalamiento oficial (Mora, 2017, febrero 22).

Pero también, en términos académicos, desde inicios del año 2016 se desarrolla la investigación denominada Construcción de un modelo interpretativo sobre la lectura como práctica social en docentes de colegios académicos y técnicos, diurnos y nocturnos de las provincias de Cartago y Heredia (en adelante, se hará referencia a ese estudio por la siguiente abreviación de su nombre: Modelo sobre la lectura como práctica social en docentes), por parte de un equipo de trabajo conformado por las personas autoras de este artículo; trabajo cuyos resultados preliminares ofrecen una serie de valiosos insumos desde los cuales discutir sobre una parte de lo que podría denominarse el problema de la lectura como problema país, escenario en el cual, según criterio de Luis Bernal Montes de Oca, presidente de la Cámara Costarricense del Libro, se requiere: “... una labor conjunta, que sea una gestión integral del Estado, no hablo del Gobierno de turno. Precisamos de una política en la que el Estado impulse el fomento a la lectura y que esa acción surja sin banderías políticas" (citado por Mora, 2017, febrero 22, párr. 12).

Así, con el análisis de los datos recogidos en la primera fase del trabajo de campo de ese proyecto, se hizo evidente que la complejidad de un problema como la lectura y su abordaje dentro de los escenarios educativos costarricenses, suponía, en primer lugar, la reflexión crítica en torno a conceptos y perspectivas ya abordadas en estudios antecedentes para, luego, detectar tendencias que orientaran, a su vez, la hermenéutica del fenómeno.

Parte de los datos recopilados en el marco de esa investigación evidenciaron una tensión de fondo en las prácticas lectoras de los entrevistados, en términos de lo que deben leer como parte de su labor institucional y lo que leen por gusto propio; de ahí que el objetivo de este artículo es discutir sobre las relaciones entre la lectura como obligación institucional y la lectura como una actividad desarrollada por gusto propio que apuntaría a la autonomía, en la práctica lectora de ocho docentes de secundaria, quienes laboran en instituciones educativas rurales y urbanas de las provincias de Heredia y Cartago.

La selección y propuesta del tema donde se enfoca la lectura como una práctica social de sujetos concretos involucrados en espacios educativos, derivó del hallazgo de ciertas líneas temáticas que se pergeñaron tras la revisión de literatura especializada publicada en los últimos diez años, y de la detección 
de algunos vacíos en el abordaje en investigaciones desarrolladas por instancias gubernamentales y en tesis de grado y posgrado en Costa Rica.

Una primera área de investigación identificada está constituida por lo que se puede denominar como el estudio de las implicaciones pedagógicas de la lectura en espacios de educación formal.

Efectivamente, diferentes trabajos asociados al ámbito educativo costarricense, tienen como objeto de interés la lectura en instituciones educativas de primaria a partir de factores sociales que incidirían en su aprendizaje (García, 2011) o comprensión (Álvarez, 2015), la incidencia de la lectura en el aprendizaje de diferentes asignaturas (Espinoza y Bolaños, 2012) o en el rendimiento académico (Caravaca, 2013; Mora y Moya, 2013), estrategias docentes para su fomento (Arce, 2014).

En el escenario de la educación superior, la investigación efectuada por Fallas, Muñoz, Ulloa y Vargas (2014) describió las prácticas lectoras de estudiantes de diferentes facultades de la Universidad de Costa Rica, pero enfocando la indagación sobre la lectura de obras literarias entre los discentes encuestados.

Ese estudio resulta de especial interés pues en él se entiende la lectura como una práctica cultural que, de acuerdo con los planteamientos de Bourdieu que siguen los autores, se entiende como

... un proceso intersubjetivo en donde el lector o lectora se ve inscrito en lo social y no está aislado de las demás personas lectoras y no lectoras con las que convive; la interacción con ellas modifica la manera en que lee un texto. Además, los y las lectores no son ajenos al bagaje histórico de la sociedad en que viven (Fallas, Muñoz, Ulloa y Vargas, 2014, p. 22).

Si bien el estudio destaca el lugar de lo institucional como espacio que tiende a la normatividad de las prácticas culturales, incluida la lectura, los autores no logran identificar cómo las actividades lectoras concretas se ajustan o contravienen esa normalización. De ahí que el estudio muestre como sus principales resultados aspectos como la frecuencia de lectura entre los sujetos encuestados, los formatos de textos preferidos (digital o papel) y las obras y autores específicos que prefiere el estudiantado consultado.

Pero también el ámbito de indagación se ha llevado hasta el análisis la lectura como hábito individual en estudios efectuados por el Ministerio de Cultura y Juventud (Montilla y Ávalos, 2012) en los que se indaga sobre pautas de consumo cultural, entre las que se ubica la lectura, e internacionales enfocados en los hábitos de lectura entre estudiantes de educación superior en Colombia (Aponte, 2008) y de primaria y secundaria en Guatemala (Valle, 2012).

Otros estudios internacionales muestran interés en el tema de la lectura, enfatizando su enfoque como un hábito que, tal como apuntan Cornejo, Roble, Barrero y Martín (2012) es parte de la práctica común de estudiantes universitarios de la Facultad de Ingeniería de la Universidad de Buenos Aires, Argentina, pero como un hábito que el estudiantado no relaciona con su formación profesional.

Si bien no corresponde a un trabajo investigativo como tal, las reflexiones de Dubois (2011) son de especial interés por su llamado a integrar la lectura como parte sustancial de la formación permanente de los profesionales en educación:

Si la formación en la lectura y la escritura es importante para cualquier profesional, con mucha mayor razón para quienes estamos encargados de dirigir el desarrollo de esos procesos en niños, jóvenes, o adultos, es decir, para nosotros, los docentes. Nuestra formación, en este caso, tiene que abarcar, necesariamente, el saber, el hacer y el ser (p. 67).

En ese sentido, la autora ofrece una serie de cuestionamientos hacia el papel del docente como formador, sustentados en experiencias de formación en lectura y escritura impartidas en el Postgrado de 
Lectura de la Universidad de Los Andes, de Mérida, Venezuela; en donde se enfatiza el carácter interactivo, cooperativo y solidario de la formación docente, y en donde se enfoca la lectura y la escritura como procesos que se comparten con el estudiantado, es decir, que involucran una práctica dialógica y de construcción social.

En general, se puede afirmar que no hay una tradición hecha en el abordaje de la lectura como práctica social, en la que, por un lado, se indaguen sus implicaciones institucionales en el quehacer docente. La mayor parte de los trabajos reseñados se enfocan en la lectura de estudiantes de diferentes etapas educativas y aunque el supracitado análisis de Fallas, Muñoz, Ulloa y Vargas (2014) conciben la lectura como una práctica cultural, los datos y la discusión efectuada por estos autores no permite reconocer la dinámica que se establece entre la normatividad institucional y la práctica lectora concreta. Además, la preocupación en torno a la lectura como parte de la formación docente no ha sido motivo de estudios aunque sí ha motivado reflexiones como la efectuada Dubois (2011).

\section{La lectura como práctica social}

Contrario a la común imagen de la persona solitaria que lee sin prestar atención a su entorno, la actividad lectora está estrechamente asociada a la trama sociocultural de la que participa el sujeto lector. Y ese vínculo se evidencia de diferentes maneras.

Por ejemplo, si el análisis de esa relación entre las condiciones socioculturales y la lectura se centra en lo que, parafraseando a Courtés (1997), puede entenderse como un acercamiento hermenéutico a la estructura inmanente de la acción lectora, una posibilidad de problematización recaería en el tratamiento que pedagógicamente se da a los aspectos socioculturales y políticos que emergen en la decodificación semántica que supone la relación entre la persona que lee y el texto que es leído, según apunta Silveira (2013).

No obstante, la misma autora advierte que

Llegar a la lectura que va más allá de la decodificación del texto -a la alfabetización crítica con nuestros estudiantes- es un problema a solucionar. Existen dificultades en las prácticas de lectura en todos los niveles institucionales en grados diversos y con consecuencias diferentes (Silveira, 2013, p. 107).

Es decir, esa aproximación apunta a la indagación en torno a las determinaciones socioculturales que inciden en lo que comúnmente se denomina comprensión lectora, en el ámbito educativo. Sin embargo, se debe considerar que las repercusiones de lo social en la lectura no se agotarían en esa problemática inmanente, sino que la rebasan con mucho llegando, incluso, a marcar la forma cómo se lee, lo que se lee, cuándo se lee.

Así, la indagación alrededor de la lectura como práctica social se dirige a lo que Gómez (2008) identifica como el interés de una perspectiva histórico-cultural, al cuestionar más allá de la pretendida crisis del libro como canal básico:

¿no tendríamos que preguntarnos por las condiciones de posibilidad necesarias para que la lectura produzca semejantes manifestaciones? ¿Qué tipo de autores y textos? ¿Leídos en qué condiciones? ¿Por parte de qué clase de lectores o comunidades de intérpretes? Esto es, ¿lectores, cultural y socialmente configurados en relación a qué tipo de habitus respecto de la lectura y, en general, respecto de qué otras formas de consumo cultural es posible que se de ese tipo de procesos psico-sociales? (p. 13). 
En consonancia con esa perspectiva, la lectura como práctica social se puede comprender a partir del concepto de habitus de Bourdieu (2007) en tanto sistema de disposiciones que fungen como

... principios organizadores de prácticas y representaciones que pueden estar objetivamente adaptadas a su fin sin suponer la búsqueda consciente de fines y el dominio expreso de las operaciones necesarias para alcanzarlos, objetivamente reguladas y regulares sin ser el producto de reglas, y a la vez que todo esto, colectivamente orquestadas... (p. 86).

Según ese punto de vista, la lectura es una forma de consumo cultural que, pautada por el habitus, estaría regida por principios y representaciones que hacen de la persona lectora un sujeto cuyos objetivos de lectura pueden pasar desapercibidos, en tanto han sido incorporados a modo de formas casi natural de su actividad cotidiana.

Es por ello que ubicar la lectura como práctica social, e históricamente delimitada, supone interrogar la naturalidad con que se realiza en tanto está arraigada como un hábito personal cuyo apuntalamiento está dado por la estructura social. Así, según Bourdieu (citado por Silva, 2003)

Si es cierto lo que afirmo, que la lectura es el producto de las condiciones en las cuales alguien ha sido producido como lector, tomar conciencia de ello es la única manera de escapar al efecto de esas condiciones, lo que otorga una función epistemológica a toda reflexión histórica sobre la lectura (p. 164).

En ese sentido, la lectura como práctica estará conformada por disposiciones y lógicas cuyo origen está en la estructura social y se materializa en las relaciones dentro de las instituciones tanto de la persona que lee con los textos, como de esta con otros sujetos lectores cuya actividad puede tender a la reproducción de lo instituido o a la tensión respecto de esa constricción.

Por ello, se entiende que la lectura institucionalizada es una forma de práctica lectora caracterizada, según lo anotado por Ulloa (2017), por constricciones institucionales que marcan la historia grupal o individual de las biografías lectoras, en cuanto a normas y formas de leer que pueden asociarse con deberes escolares, laborales o de otras agrupaciones institucionales en las que participa el sujeto (grupos religiosos, políticos o artísticos) en tanto estos determinen la forma de leer y los textos que se leen, a partir de criterios que, como apunta Bourdieu (2010), se instituyen "... como capacidad de considerar en sí y por sí mismas, en su forma y no en su función, no solo las obras designadas por tal aprehensión, es decir, las obras de arte legítimas, sino todas las cosas del mundo..." (p. 235).

Mientras que la lectura como autonomía se identifica con la búsqueda de textos y el seguimiento de criterios y normas no institucionales (aunque pueda darse dentro de escenarios institucionales), y que involucraría actividades individuales o grupales que apunten a "... soluciones de ruptura, más o menos radical, respecto de esa institucionalidad" (Ulloa, 2017, p. 6). Si la lectura como autonomía sucede dentro de escenarios institucionales podría adquirir las dimensiones de una tensión respecto de las constricciones institucionales y puede estar asociada a agrupaciones más o menos estables.

Los resultados e interpretaciones que se exponen a continuación son parte del proceso de reflexión y detección de tendencias categoriales, y responden al objetivo de discutir parte de los hallazgos que configuran las relaciones entre la lectura como obligación institucionalizada y la lectura como práctica que puede apuntar a la autonomía, según la práctica lectora de los ocho docentes de secundaria. 


\section{METODOLOGÍA}

El proceso investigativo que orientó el proyecto Modelo sobre la lectura como práctica social en docentes, de donde se derivan los datos que aquí se discuten, se efectuó dentro del paradigma naturalista, enfocado desde el modelo cualitativo, por cuanto la información se recolectó con el interés de comprender e interpretar los diversos aspectos relacionados con la práctica de la lectura en los educadores que participaron de la investigación.

Además, aquella investigación base se desarrolló bajo la modalidad de teoría fundamentada (Glasser y Strauss, 1999; Strauss y Corbin, 2002), por lo cual los nuevos conocimientos de orden teórico sobre la actividad lectora en las personas educadoras entrevistadas para ese proceso investigativo, dieron lugar a una serie de categorías analíticas que, en parte, se retoman e interpretan aquí a la luz de los planteamientos teóricos enunciados en el apartado anterior, sobre la lectura como práctica social y la tensión entre lo institucional y la autonomía.

En ese sentido, metodológicamente se apostó por la posibilidad de recolectar información cualitativa que, más allá de la medición de hábitos, permitiera identificar trayectorias vitales de lectura y formas de consumo de textos en aras de la construcción de categorías emergentes enfocadas en la interpretación de las prácticas lectoras de las personas docentes entrevistadas.

Precisamente la tensión entre lo institucional y la autonomía como parte de la actividad lectora formó parte de las categorías emergentes del estudio base, configurándose como un núcleo conceptual significativo cuyo abordaje específico sustenta lo expuesto en este artículo.

\section{Sujetos y contexto}

Los datos que se exponen y discuten aquí fueron recabados durante la primera fase del trabajo de campo del proyecto Modelo sobre la lectura como práctica social en docentes, fase en la que se entrevistó a ocho docentes de secundaria, de los cuales cuatro eran de instituciones públicas de la provincia de Cartago y cuatro de la provincia de Heredia, en zonas urbanas y rurales.

La selección de los sujetos se basó en los siguientes criterios:

- Experiencia de al menos 5 años de docencia en instituciones de educación secundaria.

- De esos 5 años de experiencia, al menos 3 debían de ser en la institución de la provincia de Heredia o Cartago donde labora actualmente.

- Laborar en una jornada de al menos medio tiempo (20 lecciones) en la institución de la provincia de Heredia o Cartago donde trabaja actualmente.

- Ser docente de cualquiera de las asignaturas básicas o de las complementarias.

La distribución de docentes según asignatura, provincia y zona donde se ubica la institución educativa se presenta en el siguiente tabla: 
TABLA 1

Sujetos participantes en la primera fase del trabajo de campo del estudio Modelo sobre la lectura como práctica social en docentes

\begin{tabular}{clllc}
$\begin{array}{c}\text { Cantidad de } \\
\text { docentes } \\
\text { entrevistados }\end{array}$ & \multicolumn{1}{c}{$\begin{array}{c}\text { Asignaturas } \\
\text { que imparten }\end{array}$} & Nombre de la institución & Provincia & Zona \\
\hline 2 & Español y Orientación & Colegio Humanístico Costarricense & Heredia & Urbana \\
2 & Educación Física e Informática & Liceo Ambientalista de Horquetas de Sarapiquí & Heredia & Rural \\
\hline 2 & Español y Estudios Sociales & Liceo Experimental Bilingüe José Figueres Ferrer & Cartago & Urbana \\
\hline 2 & Ciencias y Matemáticas & Liceo de Corralillo de Cartago & Cartago & Rural
\end{tabular}

Fuente: elaboración propia.

La elección de estas instituciones se basó en el criterio de conveniencia, pues se facilitó el contacto y permisos correspondientes con el personal administrativo y docente de esos recintos educativos, y en ellos se cumplía con los requerimientos técnicos del estudio.

\section{Técnicas para la recolección de información}

Se empleó la técnica de la entrevista narrativa-biográfica según la propuesta de Apitzsch \& Siouti (2007), adaptada en términos de un cuestionario que tenía como base un conjunto de interrogantes generadoras que facilitaran la reconstrucción de la biografía de las personas docentes entrevistadas, enfatizando las prácticas concretas de lectura de cada uno, instrumento que se validó por medio de su revisión por parte de expertos.

\section{Procedimiento analítico}

Para esta ponencia los datos derivaron del proceso de análisis efectuado en el estudio Modelo sobre la lectura como práctica social en docentes, tomando como base las categorías emergentes denominadas Lectura institucional y Lectura como autonomía, las cuales se conceptualizaron posteriormente siguiendo los planteamientos de Bourdieu (2007) y Ulloa (2010), según puede verse en el siguiente tabla: 
TABLA 2

Categorías para el análisis de los datos

\section{Categorías Definición}

Lectura Consiste en la práctica lectora caracterizada, según lo anotado por Ulloa (2017), por constricciones

institucional institucionales que marcan la historia grupal o individual de las biografías lectoras, en cuanto a normas y formas de leer que pueden asociarse con deberes escolares, laborales o de otras agrupaciones institucionales en las que participa el sujeto (grupos religiosos, políticos o artísticos) en tanto estos determinen la forma de leer y los textos que se leen, a partir de criterios que, como apunta Bourdieu (2010), se instituyen “... como capacidad de considerar en sí y por sí mismas, en su forma y no en su función, no solo las obras designadas por tal aprehensión, es decir, las obras de arte legítimas, sino todas las cosas del mundo..." (p. 235).

Lectura como Consiste en una práctica lectora que se identifica con la búsqueda de textos y el seguimiento de criterios autonomía y normas no institucionales, y que puede darse como parte de actividades individuales o grupales que apunten a "... soluciones de ruptura, más o menos radical, respecto de esa institucionalidad" (Ulloa, 2017, p. 6). La lectura como autonomía podría darse dentro de escenarios institucionales como una tensión respecto de las constricciones institucionales y pueden estar asociadas a agrupaciones más o menos estables.

Fuente: elaboración propia.

En todo caso, la lectura se da como una práctica que, de acuerdo con la biografía del sujeto, puede estar marcada por momentos de institucionalidad y de autonomía, por lo que el análisis de las trayectorias particulares debe dar cuenta de la manera como se articula la tensión entre una y otra forma de leer.

Con base en ambas categorías se ordenaron los datos que permitían su saturación y se elaboraron nuevas categorías que permitieran sintetizar rasgos más específicos dentro de cada una de esas prácticas lectoras.

\section{RESULTADOS}

En general, el inicio en la práctica lectora de las personas docentes entrevistadas tuvo como escenario la escuela y lo que en ella se enseñaba respecto a la lectoescritura, aunque en algunos casos se trató de una confluencia entre el apoyo familiar y la institución educativa, como puede verse en la siguiente tabla sintética: 
TABLA 3

Institucionalidad en el inicio de la práctica lectora

\section{Subcategoría Segmento de entrevista'}

Escuela como espacio de aprendizaje exclusivo

escuela y familia

Confluencia
E1LJFF: Yo comencé a leer más o menos como a los 7 años o 6 y medio, entre a los 6 años y medio a la escuela y rápido empecé aprendí a leer.

$P$ : Quién le enseño a leer

E1LJFF: Bueno la verdad en la escuela

E2LAHS: a los 7 años cuando entre a la escuela

$P:$ a los 7 años OK

$P$ : como fueron esas primeras experiencias con la lectura

E2LAHS: no muy buenas

E2LCC: ajá...bueno cuando tenía más o menos cinco años...cuando ya ingresé no al kínder sino antes se le llamaba pre kínder verdad y este... recuerdo que fue porque mi papá tenía varios libritos de cuando él estaba en la escuela los tenía guardados entonces me gustaba revisarlos porque eran digamos libros atractivos... tenían dibujillos de colores...si ahí fue cuando me intereso aprender a leer de hecho lo hice antes de ingresar a la escuela

E2CHC: bueno cuando yo estaba en la escuela habían unos libritos Ilamados hacia la luz... más o menos de ellos fue de donde yo aprendí a leer...también tenía que mi mamá me compraba libros adicionales...el Silabario Castellano... y ese de quien era... de Porfirio algo se llamaba el autor no recuerdo

Familia como E2LJFF: No recuerdo la edad exactamente, pero yo antes de entrar a la escuela ya sabía leer mi mama me ayudaba a aprender palabras, letras también me contaba cosas

Fuente: elaboración propia.

En las entrevistas realizadas se evidencia cómo tanto la institucionalidad escolar como familiar son los espacios en los que los primeros acercamientos a la lectura tienen lugar, situación que no resulta novedosa pero que a efectos de este análisis permite afirmar que la práctica lectora está, estructuralmente, asida a las instituciones socializadoras y estaría, tal como plantea Bourdieu (2010), regida por los criterios de selección y distinción que desde la primera infancia ambos escenarios inculcan en el habitus del sujeto lector.

Ello significa que los valores, criterios y normas sobre la lectura que inicialmente marcaría la práctica lectora de las personas docentes entrevistadas, emergen como incorporaciones de lo que puede denominarse una práctica institucionalizada ante la cual, sin embargo, la persona lectora puede desarrollar acciones que apunten hacia formas de lectura autónomas que complementarían tal normatividad.

Era común que esa tensión se expresara, según el testimonio de las personas docentes entrevistadas, dentro de los recintos educativos en un forzado cumplimiento de las lecturas obligatorias combinadas con aquellas búsquedas de textos cuyos temas parecían de exclusivo interés personal, tal como puede verse en la siguiente tabla:

1 En los segmentos de entrevistas se identifica a las personas entrevistadas por medio de códigos como E2LJFF, los cuales indican el número de entrevista y la institución donde se realizó. La palabra "P" se emplea para identificar las intervenciones de quien aplicó la entrevista. 
TABLA 4

Oposición entre la normativa institucional y la lectura autónoma en la formación profesional

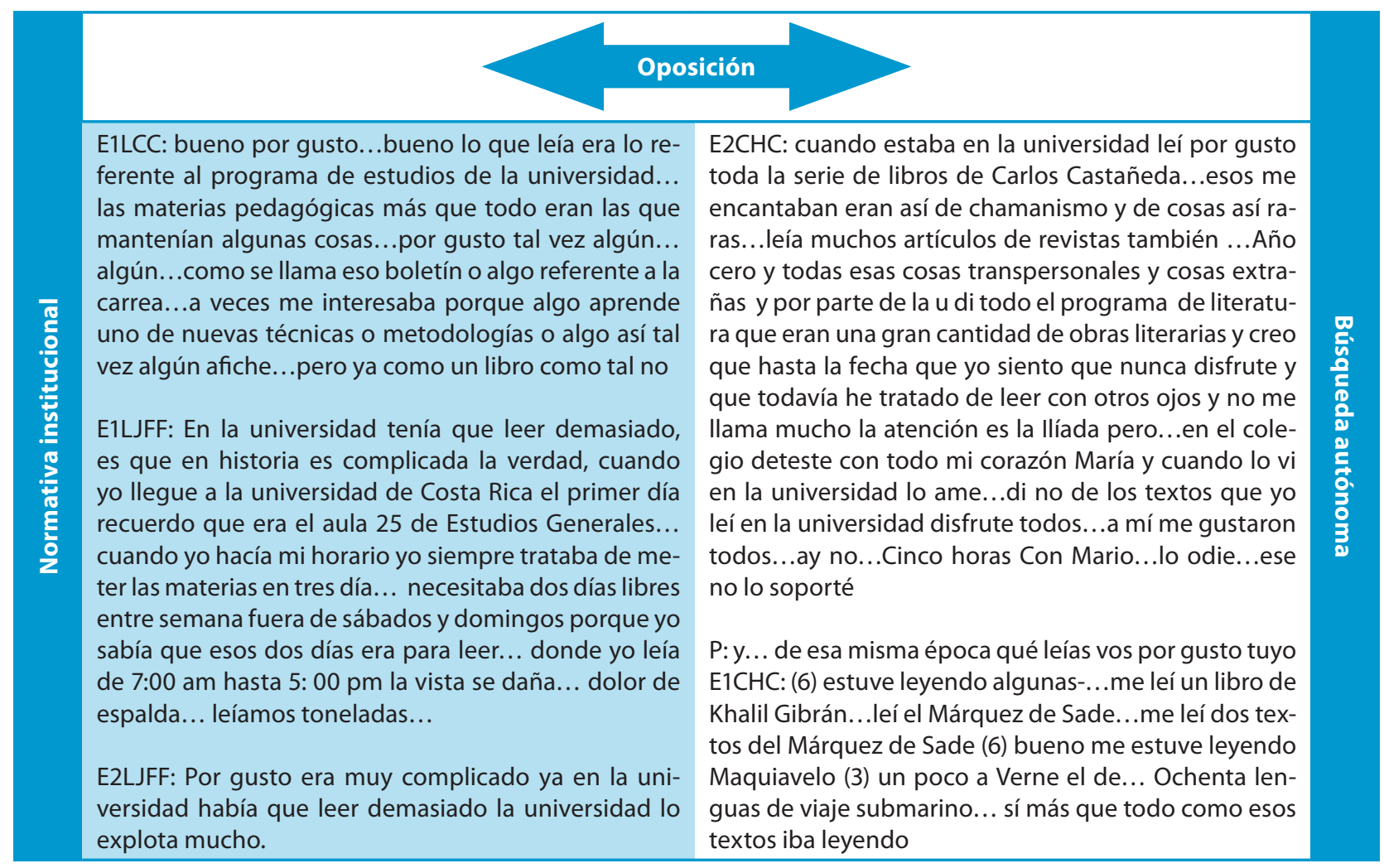

Fuente: elaboración propia.

En ese sentido, la oposición entre lo normativo institucional y la búsqueda de una práctica lectora autónoma se expresa en la tensión de fondo que experimentaban los docentes consultados durante su formación universitaria.

Se trataría de una pugna entre la obligatoriedad de la lectura que, amén del agrado o no por lo leído, se convertía en una suerte de lucha por la sobrevivencia académica en la que la posibilidad de leer textos ajenos a lo institucionalmente establecido podría verse obstaculizado por lo que uno de los entrevistados denominó la explotación de la universidad.

Esa oposición, que en el discurso de las personas docentes entrevistadas no fue significada en términos de complementariedad entre una práctica lectora adherida principalmente a los requerimientos institucionales y la lectura autónoma, se expresaría en el actual quehacer de los docentes entrevistados como una forma de práctica que se puede denominar como tendencia a la lectura para el trabajo, es decir, una forma de práctica lectora que corresponde a la demandas del espacio profesional y en la que, poco a poco la tendencia a leer por gusto propio iría cediendo, tal como puede verse en los siguientes segmentos discursivos: 
TABLA 5

Tendencias en la práctica lectora actual de las personas docentes consultadas

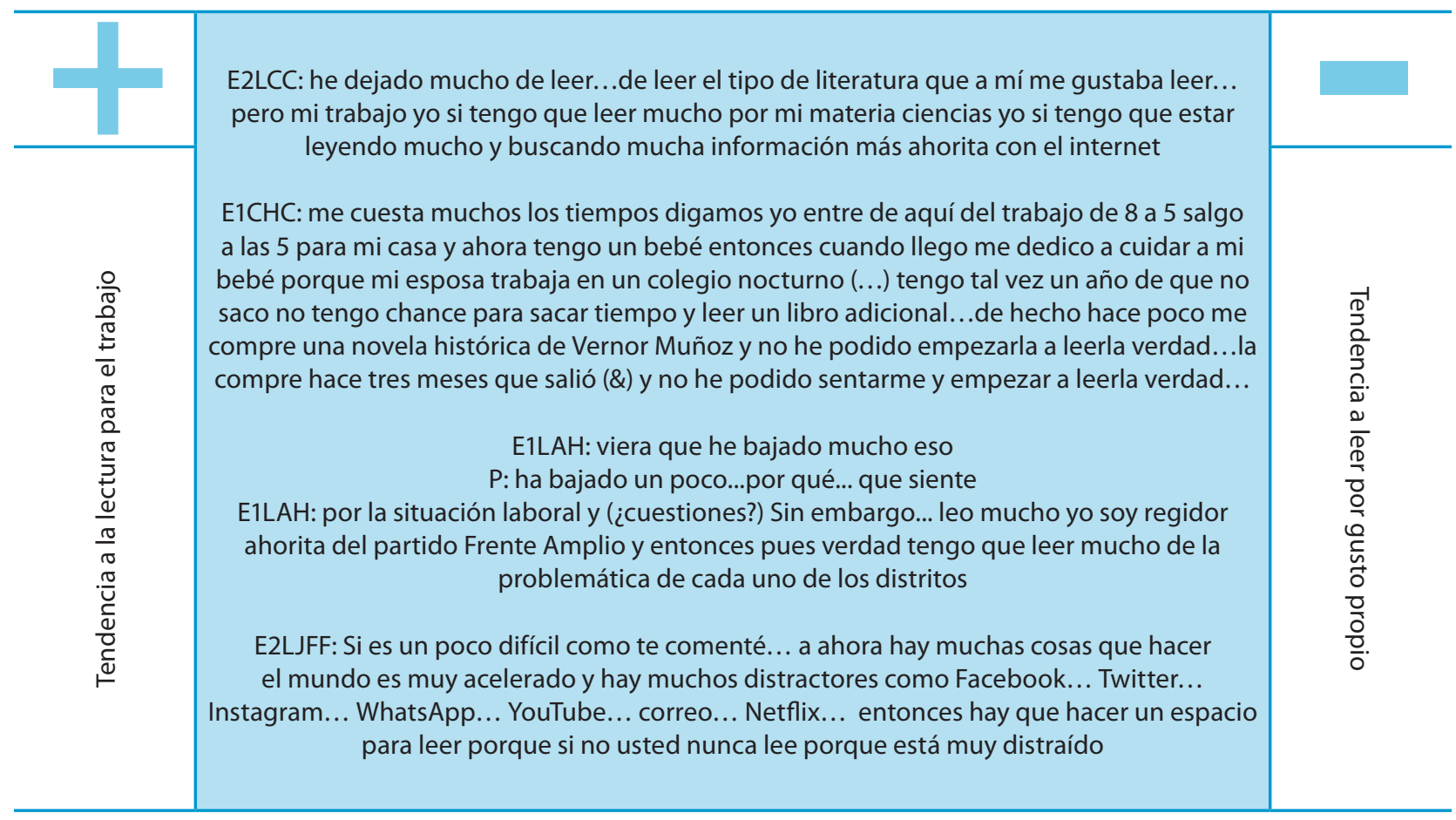

Fuente: elaboración propia.

Vale aclarar que la tendencia a la institucionalización lectora, según se ve en el testimonio de las personas docentes entrevistadas, se puede entender incluso como una estrategia para sobrevivir ante las múltiples demandas que los sujetos lectores deben atender tanto en el espacio laboral como su cotidianidad personal, aunando a ello las nuevas responsabilidades derivadas de las tecnología de la información y la comunicación.

Ante eso, parece que las personas docentes optan por centrar sus esfuerzos de lectura alrededor de aquello que, en primera instancia, permita cumplir con las demandas instituidas en el contexto laboral, lo cual es valorado de manera positiva si se entiende que de una forma que es entendida como natural la lectura se inclina hacia o se limita por el cumplimiento de deberes laborales o hasta familiares, dejando en una especie de segundo plano, la práctica lectora basada en un interés más bien autónomo.

\section{DISCUSIÓN}

A partir de los resultados expuestos en el apartado anterior, es posible identificar una efectiva oposición en el discurso de las personas docentes entrevistadas, entre la lectura como una práctica anclada en las constricciones institucionales, frente a la posibilidad de leer de forma autónoma, buscando texto por gusto propio.

En ese sentido se puede señalar que se trataría de una oposición que, lejos de incapacitar por completo la práctica lectora, se puede entender a modo de una solución de compromiso en la cual lo obligatorio, que gana más tiempo y compromiso de parte de la persona docente, convive con al menos la intención siempre presente de leer por gusto propio. 
De ahí que el gusto propio pueda entenderse como la contradicción de una práctica en la que la lectura realizada por obligación laboral (o escolar en su momento), copa factores subjetivos como la representación del tiempo (no tener tiempo para leer) y la de la información (mucha información que distrae), configurando lo que se puede entender como un gusto apropiado que, finalmente, se convertiría en núcleo valorativo de la práctica lectora, cuando la lectura normada institucionalmente se naturaliza como una oposición al gusto propio.

Y es que, efectivamente, se trata de una conversión de la necesidad en virtud, es decir, un componente del habitus, por la cual la regulación institucional misma respalda la eficacia de la práctica dentro de un determinado campo social, en este caso, el educativo, haciendo de la norma algo normal.

Así, los datos obtenidos hasta el momento permiten reflexionar acerca de los espacios que las personas participantes tienen para leer por un gusto apropiado que, quizá, normaliza la obligación institucional con el poco frecuente espacio para la lectura por gusto propio, pues, efectivamente, la mayoría de los sujetos manifiestan que gran parte de la lectura que realizan se relaciona con sus quehaceres laborales, en el tanto, deben leer para desarrollar los contenidos y temas de los programas de estudio de las asignaturas.

En este sentido, y desde lo planteado por Bourdieu (2007) la lectura como práctica social sufre una serie de constricciones institucionales que le impiden al sujeto leer por gusto, y abocarse mayormente a las lecturas escolares, laborales o de los grupos sociales donde participe.

Las personas docentes entrevistadas señalaron también que la institución escolar fue el espacio donde las primeras experiencias en la lectura se configuraron institucionalmente, aunque fuera de este ámbito mencionaron lo familiar como lugar donde padres o madres jugaron un papel muy importante, que vino a complementar los procesos escolares.

Tal y como se evidencia en los resultados, al analizar la relación entre la oposición de la normativa institucional y la búsqueda de la autonomía en la lectura, los participantes manifiestan tener pocos espacios para una práctica lectora autónoma, pues desde la escuela, el colegio, la universidad y luego en su inserción laboral, la lectura tiende a centrase en lo establecido institucionalmente, aunado a otros distractores, según plantea una de las personas docentes entrevistadas, como las redes sociales, la televisión o el teléfono móvil.

Entonces, si bien se puede afirmar que en esta disyuntiva prevalece una práctica lectora arraigada a las constricciones institucionales (a expensas del gusto propio), uno de los participantes manifestó que algunas lecturas obligatorias durante su época de colegio no le gustaban pero que en la universidad debió volver a leerlas y tuvo una experiencia enriquecedora; claro está, la formación, el bagaje cultural individual y social es diferente, e influye en su cosmovisión. Inclusive, es oportuno mencionar que diversos factores pudieron impedir que en la época escolar esas lecturas obligatorias o institucionales fueran de su agrado, como el abordaje metodológico, el dominio del tema por parte del docente, la explicación sociohistórica de la temática, entre otros.

\section{CONCLUSIONES}

Las posibilidades interpretativas que ofrece el material aquí analizado son muchas, por lo que las conclusiones que se presentan a continuación se pueden entender como aseveraciones parciales sobre el tema, las cuales pueden animar la realización de futuras investigaciones. 
En primer lugar, se puede concluir que en la práctica de los sujetos participantes domina una forma de lectura que tiende a normalizar las constricciones propias del espacio laboral, convirtiéndolas en pautas que naturalizan la actividad lectora marcada fundamentalmente por los requerimientos institucionales.

Es significativo, en ese sentido, que el primer acercamiento de las personas con la lectura (y en particular con los libros como medio principal de la actividad lectora) suele tener como centro la conjunción dada entre la familia y la escuela; y que el ingreso a la institución educativa marca en buena medida trayectorias lectoras que, al tenor de las asignaciones escolares, se configuran a partir de los requerimientos que hacen que la necesidad de leer lo que es obligatorio, se convierta en una especie de virtuoso seguimiento de lo instituido, aunque se atisben espacios para una actividad lectora proclive a la autonomía, sin que ello redunde en una total ruptura respecto de la obligatoriedad instituida.

Desde ahí se puede interrogar cómo el propio capital cultural de padres y madres de familia, también es un aspecto que puede influir en la constricción misma que desde lo institucional marca la práctica lectora, en tanto se entiende como un capital cuyo destino inquebrantable es su inversión en la escuela.

Por otro lado, sin embargo, es llamativo que se externe cierta desazón por la falta de tiempo para leer por gusto propio, pero lo es más por el hecho de que esta carencia percibida se entienda como una especie de menoscabo a la posibilidad de leer realmente. Es decir, al parecer, una constante a lo largo de la trayectoria lectora de las personas docentes entrevistadas es la de la paulatina separación entre lo que podría denominarse una forma de lectura plena, es decir, aquella que se practica en acuerdo con los intereses más personales, y una lectura que no permite leer, o sea, la que se efectúa por el cumplimiento de ciertos órdenes institucionales.

No obstante, como se indicó antes, la trayectoria lectora de las personas docentes entrevistadas tiende a armonizar esa aparente ruptura entre un gusto propio marcado por la posibilidad de leer de forma autónoma, con las exigencias de la lectura que imperan en el ámbito laboral y que, en buena medida, no son sino prolongaciones de la obligatoriedad experimentada ya desde la inserción en la institucionalidad educativa.

Además, surge también el espacio de lo virtual, de las tecnologías como el internet en tanto fuente de información que distrae, aunque a la vez se puede constituir en repositorio utilizado para acceder a lecturas que refuerzan lo instituido. De ahí que se trate de un recurso que, en el mejor de los casos, parece ser complemento de formas de actividad lectora encaminadas a responder a los requerimientos de la institucionalidad laboral (reserva de textos útiles en las lecciones y de materiales didácticos), pero que ninguna de las personas docentes consultadas mencionó como un recurso para la lectura proclive a la autonomía.

Finalmente, la indagación efectuada provee de una perspectiva en la que la oposición entre la actividad lectora institucionalmente constreñida, y la lectura que apuntaría a la autonomía de la persona docente en tanto sujeto lector, se presenta matizada por soluciones de compromiso que tratan de convertir la práctica lectora en una actividad que se realiza, cotidianamente, por gusto apropiado, esto es, por un gusto en el que hay significativas coincidencias entre el quehacer docente y las múltiples demandas que esta inscripción social representa, y lo que se hace en aras de una acción autónoma que acaba como complemento al que se acude cuando se puede y no cuando se quiere.

\section{REFERENCIAS}

Álvarez, M. (2015). Factores académicos que influyen en la comprensión de lectura en los niños y las niñas de la sección 2-6 de la Escuela Cleto González Víquez del circuito 01 de la Dirección Regional de Heredia 
año 2013. (Tesis de Licenciatura en Ciencias de La Educación con énfasis en I y II Ciclos, Escuela de Ciencias de la Educación, UNED).

Apitzsch, U. \& Siouti, I. (2007). Biographical Analysis as an Interdisciplinary Research Perspective in the Field of Migration Studies. Frankfurt: Johann Wolfgang Goethe Universität Frankfurt am Main.

Aponte, M. (2008). Métodos, preferencias y hábitos de lectura en estudiantes de pregrado. Revista Teoría y Praxis Investigativa, 3(1), febrero.-agosto, 29-35.

Arce, D. (2014). Análisis de las estrategias implementadas para el fomento de la lectura en los niños y las niñas de II Ciclo Escolar y su efecto en el mejoramiento de la capacidad lectora y comprensiva, en la Escuela Pablo Alvarado Vargas, circuito 06, de la Dirección Regional Educativa de Occidente, durante el curso lectivo 2013. (Tesis de Licenciatura en Ciencias de La Educación con énfasis en I Y II Ciclo, Escuela de Ciencias de la Educación, UNED).

Bourdieu, P. (2007). El sentido práctico. Buenos Aires: Siglo XXI.

Bourdieu, P. (2010). El sentido social del gusto. Elementos para una sociología de la cultura. Buenos Aires: Siglo XXI.

Caravaca, H. (2013). Incidencia de la lectura en el rendimiento académico en los niños y niñas de segundo ciclo que participan en el programa de recuperación integral(PRIN), de la Escuela Bernardo Gutiérrez, circuito 06, Dirección Regional de Educación de Santa Cruz, durante el periodo 2012. (Tesis de Licenciatura en Ciencias de La Educación con énfasis en I Y II Ciclo, Escuela de Ciencias de la Educación, UNED).

Cornejo, J., Roble, M., Barrero, C. \& Martín, A. (2012). Hábitos de lectura en alumnos universitarios de carreras de ciencia y de tecnología. Revista Eureka sobre Enseñanza y Divulgación de las Ciencias. 9(1), 155-163.

Courtés, J. (1997). Análisis semiótico del discurso. Del enunciado a la enunciación. Madrid: Gredos.

Dubois, M. (2011). La lectura en la formación y actualización del docente. Comentario sobre dos experiencias. Legenda. 15(12), 65-79.

Espinoza, E. \& Bolaños, F. (2012). Los procesos de enseñanza de la lectura y su influencia en el aprendizaje de otras asignaturas de los niños, en las Escuelas Unidocentes y en las Escuelas Técnicas del Circuito 03 de la Dirección Regional de Liberia. (Proyecto de graduación de Licenciatura en Ciencias de La Educación con énfasis en I Y II Ciclo, Escuela de Ciencias de la Educación, UNED).

Fallas, M., Muñoz, R., Ulloa, S. \& Vargas, L. (2014). Prácticas de lectura de novelas literarias en estudiantes de la Universidad de Costa Rica. Revista Reflexiones. 93(1), 19-32.

García, C. (2011). Análisis de los factores metodológicos, familiares, económicos y de dificultad en el aprendizaje que inciden en el desarrollo de la enseñanza de la lectura en las escuelas de Matapalo y Tres Amigos del Circuito Escolar 02 de la Dirección Regional Cañas. (Proyecto de graduación de Licenciatura en Ciencias de La Educación con énfasis en I Y II Ciclo, Escuela de Ciencias de la Educación, UNED).

Glasser, B. \& Strauss, A. (1999). The Discovery of Grounded Theory: Strategies for Qualitative Research. New York: Aldine De Gruyter.

Gómez, M. (2008). La lectura desde una perspectiva histórico-cultural. La lectura como práctica social. Revista NEXUS Comunicación. (4), julio-diciembre, 9-36.

Mora, J. (2017, febrero 22). País reclama una política nacional del libro y la lectura. Semanario Universidad. Recuperado de http://semanariouniversidad.ucr.cr/cultura/ pais-reclama-una-politica-nacional-del-libro-la-lectura/

Mora, H. y Moya, M. (2013). Propuesta de estrategias de Lectura Comprensiva para mejorar el rendimiento académico en las asignaturas de Ciencias y Estudios Sociales, del estudiantado de IV y V Grados de la Escuela La Suiza, Circuito 07 de la Dirección Regional de Educación de Pérez Zeledón, año 2012. (Tesis de Licenciatura en Ciencias de La Educación con énfasis en I y II Ciclo, Escuela de Ciencias de la Educación, UNED). 
Montilla, A. y Ávalos, C. (2012). Análisis de la Encuesta de prácticas y hábitos culturales de Costa Rica, 20102011. San José: Dirección de Cultura, Ministerio de Cultura y Juventud.

Silva, R. (2003). La lectura: una práctica cultural. Debate entre Pierre Bourdieu y Roger Chartier. Revista Sociedad y Economía, (4), abril, 161-175.

Silveira, E. (2013). La lectura como práctica sociocultural y herramienta para lograr la equidad social a partir de la enseñanza. Discusión acerca la enseñanza de la lectura. Cuadernos de Investigación Educativa, 19(4), 105-113.

Strauss, A. y Corbin, J. (2002). Bases de la investigación cualitativa. Técnicas y procedimientos para desarroIlar la teoría fundamentada. Medellín: Editorial Universidad de Antioquia.

Ulloa, G. (2017). Construcción de categorías analíticas para el estudio de las prácticas lectoras. Ponencia presentada en el XIV Congreso Latinoamericano para el Desarrollo de la Lectura y Escritura. 28 al 30 de setiembre, San José, Costa Rica. Organizado por Universidad de Costa Rica, Universidad Nacional, Asociación Costarricense Consejo de Lectura, International Literacy Association y Comité Latinoamericano para el Desarrollo de la Lectura y la Escritura.

Valle, M. (2012). Variables que inciden en la adquisición de hábitos de lectura de los estudiantes. Ciudad de Guatemala: Dirección General de Evaluación e Investigación Educativa. 